\title{
Authors' reply re: Reflex cytology for triage of high-risk human papillomavirus positive self-sampled material in cervical cancer screening: a prospective cohort study. (Response to BJOG-20-1479)
}

\author{
Diede Loopik ${ }^{1}$, Willem Melchers ${ }^{2}$, Judith Vedder ${ }^{2}$, Adriaan van den Brule ${ }^{3}$, Leon \\ Massuger $^{2}$, Ruud Bekkers ${ }^{4}$, and Bert Siebers ${ }^{2}$ \\ ${ }^{1}$ Affiliation not available \\ ${ }^{2}$ Radboudumc \\ ${ }^{3}$ Jeroen Bosch Hospital \\ ${ }^{4}$ Catharina Hospital
}

July 27, 2020

Dear editor/Sir,

We thank Song et al. for their insightful comments (1) on our article (2) and commend them for their study about human papillomavirus (HPV)-genotyping on self-samples and their analysis on different triage strategies for detecting cervical intraepithelial neoplasia grade 2 or worse (CIN2+) (3). They report that cytology on physician-sampled material has a sensitivity of $74.8 \%$ for detecting CIN2+, while HPV-genotyping for $16 / 18$ has a sensitivity of $52.6 \%$ for detecting CIN2+. This means that HPV-genotyping in their hands, is still inferior to cytology testing and cannot fully replace this triage strategy. However, adding HPVgenotyping to cytology testing could increase the sensitivity, as has been described by others as well. While our study shows that reflex cytology on self-samples cannot replace triage with regular cytology for HPVpositive women, because of the low sensitivity of $29.4 \%$ for detecting CIN2+, it is valuable as an additional method for triage (2). With a positive predictive value (PPV) of $68.1 \%$ for detecting CIN2+ it is effective to refer HPV-positive women with abnormal cytology on self-sampling directly for colposcopic evaluation without the requirement of an extra visit to the general practitioner. A PPV of $21.2 \%$ for HPV-genotyping for $16 / 18$ on self-samples for detecting CIN2+ is not enough to refer these women directly for colposcopy. We agree that cost-effectiveness is important. It is not sure if $15 \%$ of direct referral will completely cover the costs for $85 \%$ of double cytology testing. As the collection in PreservCyt (Cytyc Corporation, Boxborough, MA, USA) has already been performed for HPV-testing on the self-samples, extra costs will include the use of ThinPrep slides (Hologic Inc, Marlborough, MA, USA) and cytotechnicians' time for analysing the slides. On the other hand, there will be a reduction in costs for consulting the general practitioner and for regular cytology testing, including material costs (Cervex brush (Roversß) Medical Devices B.V., Oss, the Netherlands), PreservCyt jar, ThinPrep slide), transportation costs, and cytotechnicians' time for analysing the slides. However, besides cost-effectiveness, patient comfort is at least as important, as well as reduction in loss-to-follow-up and diagnostic delay, in which the latter also positively influences the costs. It remains a challenge to find a triage method on HPV-positive self-samples which could fully replace regular cytology. Molecular tests, such as methylation markers and microRNA detection, are promising future triage methods $(4,5)$. They are more objective than cytology testing and highly reproducible, however not ready yet for full implementation in cervical cancer screening. Further research on self-samples is warranted to find an optimal triage strategy. Until then, reflex cytology on self-samples could be easily implemented in the current screening programme and improve cervical cancer prevention. 
Diede L Loopik ${ }_{1}$; Willem JG Melchers 2 ; Judith EM Vedder ${ }_{3}$; Adriaan JC van den Brule 4 ; Leon FAG Massuger $_{1}$; Ruud LM Bekkers 5 ; Albert G Siebers 3,6

${ }_{1}$ Department of Obstetrics and Gynaecology, Radboud Institute for Molecular Life Sciences, Radboud university medical center, PO Box 9101, 6500HB, Nijmegen, the Netherlands; 2 Department of Medical Microbiology, Radboud university medical center, PO Box 9101, 6500HB, Nijmegen, the Netherlands; 3 Department of Pathology, Radboud university medical center, PO Box 9101, 6500HB, Nijmegen, the Netherlands; 4 Department of Pathology, Lab for Molecular Diagnostics, Pathologie-DNA, Jeroen Bosch Hospital, PO Box 90153, 5200ME,'s-Hertogenbosch, the Netherlands; 5 Department of Obstetrics and Gynaecology, Catharina Hospital, PO Box 1350, 5602ZA, Eindhoven, the Netherlands; 6 PALGA, the nationwide network and registry of histo- and cytopathology, Randhoeve 225a, 3995 GA, Houten, the Netherlands

Present address Ruud LM Bekkers: GROW, School for Oncology \& Developmental Biology, Maastricht University Medical Centre, PO Box 616, 6200MD, Maastricht, the Netherlands

\section{References}

1. Song F, Yan P, Wu R. Re: Reflex cytology for triage of high-risk human papillomavirus positive self-sampled material in cervical cancer screening: a prospective cohort study. Cytology on hrHPV-positive self-samples: challenges and potential solutions. BJOG: An International Journal of Obstetrics \& Gynaecology. 2020.

2. Loopik DL, Melchers W, Vedder J, van den Brule A, Massuger L, Bekkers R, et al. Reflex cytology for triage of high-risk human papillomavirus positive self-sampled material in cervical cancer screening: a prospective cohort study. Bjog. 2020.

3. Song F, Du H, Wang C, Huang X, Wu R. The effectiveness of HPV16 and HPV18 genotyping and cytology with different thresholds for the triage of human papillomavirus-based screening on self-collected samples. PLoS One. 2020;15(6):e0234518.

4. Snoek BC, Verlaat W, Babion I, Novianti PW, van de Wiel MA, Wilting SM, et al. Genome-wide microRNA analysis of HPV-positive self-samples yields novel triage markers for early detection of cervical cancer. Int J Cancer. 2019;144(2):372-9.

5. Verhoef VM, Bosgraaf RP, van Kemenade FJ, Rozendaal L, Heideman DA, Hesselink AT, et al. Triage by methylation-marker testing versus cytology in women who test HPV-positive on self-collected cervicovaginal specimens (PROHTECT-3): a randomised controlled non-inferiority trial. Lancet Oncol. 2014;15(3):315-22.

Disclosure of interest: the authors report no conflicts of interest 